Proceedings of the 50th Hawaii International Conference on System Sciences | 2017

\title{
Introduction to Cross-Organizational and Cross-Border IT/IS Collaboration Minitrack
}

\author{
Ilsang Ko \\ Chonnam National University \\ isko@chonnam.ac.kr
}

\author{
Daniel Beimborn \\ Frankfurt School of Finance \& Management \\ d.beimborn@fs.de
}

Smart devices and network intelligence with the Internet of Things (IoT) are expected to exploit crossorganizational and cross-border IT/IS collaboration in the near future. Integration of people, systems, processes, and infrastructure across organizations, borders, and world regions enables productive teamwork towards achieving mutual goals. Collaborators are motivated to enter into collaborative projects and interactions, which lead to satisfaction and performance. Quality in collaboration is fostered by perception of value, trust, and commitment among participants and stakeholders. With progressing globalization, many of these collaborations are conducted across widely dispersed organizations and national borders. Cross-system integration and collaboration technologies play crucial roles and often decide about investment success or failure. Growth in electronic and virtual collaboration can be facilitated by organizations seeking to gain competitive advantage by lowering costs, increasing knowledge, and reaching new customers.

During the last ten years from 2005 to 2014, James Pick, Nicolas Romano, and Narcyz Roztocki have served as our mini-track chairs. I and Daniel would like to express special thanks to them for their great contributions on our COCB Collaboration mini-track.

In our Cross-Organizational and Cross-Border Collaboration session, 5 papers will be presented. Easing Cross-Border Communication: MOBILE mediated Communication and Its Framework by Kyungsub Stephen Choi and Youngsoo Kim identified seven media identifiable attributes: synchronicity (SYN), de-individuation and co-presence (DCP), accessibility readiness (ARD), cognizance of environment change (CEC), wearability-portability (WRB) modality-select (MDS) and visibility (VSB). These seven attributes significantly impact the course of mobile-mediated communication and contribute to fully understanding the mobile ecosystem upon us. Material Intelligence: Cross-Organizational Collaboration Driven by Detailed Material Data by Esko Hakanen, Ville Eloranta, Pekka Töytäri, Risto
Rajala, Taija Turunen explores the influences of the use of IoT for information sharing in the steel industry networks. Shared data have multiple uses, including optimization, integration, automatization, and adaptation of objects in their environments. Collaboration among Crowdsourcees: Towards a Design Theory for Collaboration Process Design by Navid Tavanapour and Eva A. C. Bittner gave us a deeper understanding of the interaction among crowdsourcees and provided crowdsourcers with grounding for the informed design of effective collaborative crowdsourcing processes such as Prototyping, Feedback, Revising, and Submit. CrossOrganizational Software Development: Design and Evaluation of a Decision Support System for Software Component Outsourcing by Tommi Kramer, Armin Heinzl, and Tillmann Neben suggests a decision model that evaluates technical properties of software components to support the outsourcing decision with its implications on the crossorganizational distribution of development tasks. They implemented a mobile prototype for a decision support system in order to classify all software components regarding their outsourcing applicability. Spatial Solutions for the Environmental Protection Agency "Brownfields to Healthfields" Program: Utilization of Mixed Methods to Assess Application Effectiveness and Usability by April Moreno and Sarah Osailan introduced an interesting case about the utilization of a new concept of operation, which includes participative and volunteered approaches that are addressed to include the contribution of various stakeholder groups, and to further improve planning for public health. By addressing the effectiveness and usability of the web application and spatial data contents relevant to Community Based Organizations (CBOs), they were able to serve as a bridge between the federal and corporate sectors (EPA and Redhorse Corporation) to develop an application that meets their needs. 